\title{
Properties of disjunctive domination in product graphs
}

\author{
A. Lekha ${ }^{1 *}$ and K.S. Parvathy ${ }^{2}$
}

\begin{abstract}
In this paper properties of disjunctive domination in some graph products are studied. We examine whether disjunctive domination number is multiplicative with respect to different graph products, that is, $\gamma_{2}^{d}\left(G_{1} * G_{2}\right) \geq$ $\gamma_{2}^{d}\left(G_{1}\right) \gamma_{2}^{d}\left(G_{2}\right)$ for all graphs $G_{1}$ and $G_{2}$ or $\gamma_{2}^{d}\left(G_{1} * G_{2}\right) \leq \gamma_{2}^{d}\left(G_{1}\right) \gamma_{2}^{d}\left(G_{2}\right)$ for all graphs $G_{1}$ and $G_{2}$ where $*$ denotes lexicographic, tensor, strong or Cartesian product of graphs. Some other inequalities involving disjunctive domination number of product graphs and the graphs attaining these inequalities are also given.
\end{abstract}

\section{Keywords}

Domination, disjunctive domination, disjunctive domination number, graph product. .

\section{AMS Subject Classification}

05 C69.

${ }^{1,2}$ Research Department of Mathematics, St. Mary's College, Thrissur-680020, Kerala, India.

*Corresponding author: ${ }^{*}$ alekharemesh@gmail.com, lekha.a.res@smctsr.ac.in; ${ }^{2}$ parvathy.math@gmail.com, parvathy.ks@smctsr.ac.in Article History: Received 13 October 2019; Accepted 27 December 2019

\section{Contents}

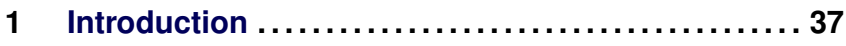

2 Preliminaries ................................. 37

3 Main Results.............................. 38

4 Conclusion $\ldots \ldots \ldots \ldots \ldots \ldots \ldots \ldots \ldots \ldots \ldots \ldots, 41$

References .................................4 41

\section{Introduction}

Various graph products clearly model processor connections in multiprocessor systems. The fast transmission of information between the processors is very important in communication systems. Hence the study of graph theoretic properties of product graphs is important. Domination number in product graphs has been studied for a long time. Among various products, the Cartesian product is the centre of study in almost all works in literature. These studies are focused largely on Vizing's conjecture. Here an attempt to determine the disjunctive domination number of different types of graph products is made.

\section{Preliminaries}

Domination in graphs is an important parameter in graph theory because of its wide applications. Tremendous research has been made by many researchers on this topic. A brilliant survey of studies related to domination is given in [2] by Haynes et al. A variation of classical domination defined as secondary dominations is studied in [3]. Another variation of domination, defined as disjunctive domination, was introduced and studied by Goddard et al. in [4]. For more details on graph products and its applications, we suggest the reader to refer [7].

Definition 2.1. A subset $S$ of the vertex set $V$ is a disjunctive dominating set or $D D$-set, if for any vertex $u \notin S$ one of the following two conditions are true.

1. there is a vertex $v \in S$ which is adjacent to $u$ or

2. there are two vertices $v_{1}, v_{2} \in S$ such that $d\left(u, v_{1}\right)=d\left(u, v_{2}\right)=2$.

The disjunctive domination number or DD-number, $\gamma_{2}^{d}(G)$ of a graph $G$ is $\min \{|S|: S$ is a $D D-$ set in $G\}[4,5]$. If the above condition is true for every vertex in $u \in S$, then $S$ is called a total disjunctive dominating set or TDD-set of $G$. Total disjunctive domination number or TDD-number, $\gamma_{t}^{d}(G)$ of $G$ is $\min \{|S|: S$ is a $T D D$ - set in $G\}$ [6].

Definition 2.2. A vertex $v$ in a graph is called a universal vertex or full degree vertex if $N[v]=V(G)$. 
Definition 2.3. A graph parameter $\phi$ is multiplicative with respect to a graph product $*$ if $\phi\left(G_{1} * G_{2}\right) \geq \phi\left(G_{1}\right) \phi\left(G_{2}\right)$ for all graphs $G_{1}$ and $G_{2}$ or $\phi\left(G_{1} * G_{2}\right) \leq \phi\left(G_{1}\right) \phi\left(G_{2}\right)$ for all graphs $G_{1}$ and $G_{2}$.

For all standard terminology and notation we follow [1]. The terms related to domonation in graphs are used as in [2].

\section{Main Results}

\section{Disjunctive domination in lexicographic products}

The Lexicographic product of graphs $G_{1}=\left(V_{1}, E_{1}\right)$ and $G_{2}=\left(V_{2}, E_{2}\right)$ is the graph $G_{1}\left[G_{2}\right]$ whose vertex set is $V_{1} \times V_{2}$ in which $\left(\left(u_{1}, v_{1}\right),\left(u_{2}, v_{2}\right)\right)$ is an edge if

- $u_{1} u_{2} \in E_{1}$ or

- $u_{1}, u_{2}$ are equal and $v_{1} v_{2} \in E_{2}$.

Theorem 3.1. Disjunctive domination number is multiplicative with respect to Lexicographic product.

Proof. Let $G_{1}=\left(V_{1}, E_{1}\right)$ and $G_{2}=\left(V_{2}, E_{2}\right)$ be graphs with $\gamma_{2}^{d}$-sets $S_{1}$ and $S_{2}$ respectively. We can show that $S_{1} \times S_{2}$ is a DD-set of $G_{1}\left[G_{2}\right]$.

\section{claim}

Let $(u, v)$ be a vertex in $G_{1}\left[G_{2}\right]$ which is not in $S_{1} \times S_{2}$.

case (i)

Let $u \in V_{1} \backslash S_{1}$ and $v \in S_{2}$. If $u$ is adjacent to $u_{1} \in S_{1}$, then $(u, v)$ is adjacent to $\left(u_{1}, v\right) \in S_{1} \times S_{2}$. If $u$ is disjunctively dominated by $u_{1}, u_{2} \in S_{1}$, then $\left(u_{1}, v\right),\left(u_{2}, v\right) \in S_{1} \times S_{2}$ and $d\left((u, v),\left(u_{1}, v\right)\right)=d\left((u, v),\left(u_{2}, v\right)\right)=2$. So $(u, v)$ is disjunctively dominated by $S_{1} \times S_{2}$.

\section{case (ii)}

Let $u \in S_{1}$ and $v \in V_{2} \backslash S_{2}$. If $v$ is adjacent to $v_{1} \in S_{2}$, then $(u, v)$ is adjacent to $\left(u, v_{1}\right) \in S_{1} \times S_{2}$. If $v$ is disjunctively dominated by $v_{1}, v_{2} \in S_{2}$, then $\left(u, v_{1}\right),\left(u, v_{2}\right) \in S_{1} \times S_{2}$ and $d\left((u, v),\left(u, v_{1}\right)\right)=d\left((u, v),\left(u, v_{2}\right)\right)=2$ so that $(u, v)$ is disjunctively dominated by $S_{1} \times S_{2}$

\section{case (iii)}

Let $u \in V_{1} \backslash S_{1}$ and $v \in V_{2} \backslash S_{2}$.

If $u$ is adjacent to $u_{1} \in S_{1}$ and $v_{1}$ is any vertex in $S_{2}$, then $(u, v)$ is adjacent to $\left(u_{1}, v_{1}\right) \in S_{1} \times S_{2}$. If $u$ is disjunctively dominated by $u_{1}, u_{2} \in S_{1}$, then $\left(u_{1}, v_{1}\right),\left(u_{2}, v_{1}\right) \in S_{1} \times S_{2}$ and $d\left((u, v),\left(u_{1}, v_{1}\right)\right)=d\left((u, v),\left(u_{2}, v_{1}\right)\right)=2$ so that $(u, v)$ is disjunctively dominated by $S_{1} \times S_{2}$.

From the above cases it follows that in each case $(u, v)$ is either dominated or disjunctively dominated by elements of $S_{1} \times S_{2}$. Thus $S_{1} \times S_{2}$ is a DD-set in $G_{1}\left[G_{2}\right]$. Hence $\gamma_{2}^{d}\left(G_{1}\left[G_{2}\right]\right) \leq \gamma_{2}^{d}\left(G_{1}\right) \gamma_{2}^{d}\left(G_{2}\right)$ for all graphs $G_{1}$ and $G_{2}$.

Remark 3.2. 1. The above bound is sharp. If $G_{1}=P_{2}$ and $G_{2}=P_{7}$, then $\gamma_{2}^{d}\left(G_{1}\right)=1, \gamma_{2}^{d}\left(G_{2}\right)=2$, $\gamma_{2}^{d}\left(G_{1}\left[G_{2}\right]\right)=$ 2, and so, $\gamma_{2}^{d}\left(G_{1}\left[G_{2}\right]\right)=\gamma_{2}^{d}\left(G_{1}\right) \gamma_{2}^{d}\left(G_{2}\right)$.
2. Strict inequality may occur in the above result. For example consider the graphs $G_{1}=P_{2}$ and $G_{2}=S_{4} \circ K_{1}$. Then $\gamma_{2}^{d}\left(G_{1}\right)=1, \gamma_{2}^{d}\left(G_{2}\right)=4, \gamma_{2}^{d}\left(G_{1}\left[G_{2}\right]\right)=2$. Here $\gamma_{2}^{d}\left(G_{1}\left[G_{2}\right]\right)<\gamma_{2}^{d}\left(G_{1}\right) \gamma_{2}^{d}\left(G_{2}\right)$.

Theorem 3.3. 1. $\gamma_{2}^{d}\left(G_{1}\left[G_{2}\right]\right)=\gamma_{2}^{d}\left(G_{1}\right)$ if $G_{2}$ has a universal vertex. In particular for a positive integer $n$, $\gamma_{2}^{d}\left(G\left[K_{n}\right]\right)=\gamma_{2}^{d}(G)$.

2. $\gamma_{2}^{d}\left(G_{1}\left[G_{2}\right]\right)=2$, if $G_{1}$ has a universal vertex, but $G_{2}$ has no such vertex. In particular, if $G_{1}=K_{n}$ and $G_{2}$ has no universal vertex, then $\gamma_{2}^{d}\left(G_{1}\left[G_{2}\right]\right)=2$.

3. If both $G_{1}$ and $G_{2}$ have a universal vertex, then $\gamma_{2}^{d}\left(G_{1}\left[G_{2}\right]\right)=1$. In particular if $G_{1}=K_{n}$ and $G_{2}=K_{m}$, where $m, n$ are positive integers, then $\gamma_{2}^{d}\left(G_{1}\left[G_{2}\right]\right)=1$.

Proof. 1. Let $v$ be a universal vertex of $G_{2}$ and $S_{1}$ be a $\gamma_{2}^{d}$ set of $G_{1}$. Then $S_{1} \times v$ disjunctively dominates $G_{1}\left[G_{2}\right]$. The minimality of $S_{1} \times v$ follows from the minimality of the $\gamma_{2}^{d}$-set $S_{1}$ of $G_{1}$. Thus, $\gamma_{2}^{d}\left(G_{1}\left[G_{2}\right]\right)=\gamma_{2}^{d}\left(G_{1}\right)$.

2. Let $u$ be a universal vertex of $G_{1}$ and $v_{1}, v_{2}$ are any two vertices in $G_{2} .\left\{\left(u, v_{1}\right),\left(u, v_{2}\right)\right\}$ forms a $\gamma_{2}^{d}$-set of $G_{1}\left[G_{2}\right]$, for if $\left(u^{\prime}, v^{\prime}\right)$ is an arbitrary vertex in $G_{1}\left[G_{2}\right] \backslash$ $\left\{\left(u, v_{1}\right),\left(u, v_{2}\right)\right\}$, then it is dominated by both $\left(u, v_{1}\right)$ and $\left(u, v_{2}\right)$ whenever $u \neq u^{\prime}$ and disjunctively dominated by $\left\{\left(u, v_{1}\right),\left(u, v_{2}\right)\right\}$ whenever $u=u^{\prime}$.

3. Let $u$ and $v$ be universal vertices in $G_{1}$ and $G_{2}$ respectively. Then $(u, v)$ dominates all the vertices in $G_{1}\left[G_{2}\right]$. So, $\gamma_{2}^{d}\left(G_{1}\left[G_{2}\right]\right)=1$.

Corollary 3.4. $\gamma_{2}^{d}\left(G_{1}\left[G_{2}\right]\right)=\gamma_{2}^{d}\left(G_{1}\right) \gamma_{2}^{d}\left(G_{2}\right)$ if $G_{2}$ has a universal vertex.

Theorem 3.5. Let $G_{1}$ be a graph without isolated vertices and $G_{2}$ be a non-trivial graph. Then,

$$
\gamma_{2}^{d}\left(G_{1}\left[G_{2}\right]\right) \leq 2 \gamma_{2}^{d}\left(G_{1}\right) .
$$

Proof. Let $S$ be a DD-set of $G_{1}$ and $x, y$ are any two distinct vertices in $G_{2}$. We can show that $(S \times x) \cup(S \times y)$ is a DD-set of $G_{1}\left[G_{2}\right]$. Clearly, $S \times x$ dominates or disjunctively dominates all the vertices in $\left(G_{1} \backslash S\right) \times G_{2}$. Now, let $(u, v)$ be a vertex in $S \times G_{2}$. Let $u^{\prime}$ be a vertex in $G_{1}$ which is adjacent to $u$ in $G_{1}$. Then $(u, v)$ is adjacent to $\left(u^{\prime}, x\right)$ which is adjacent to $(u, x) \in S \times x$ and $(u, y) \in S \times y$ in $G_{1}\left[G_{2}\right]$. It shows that every vertex in $S \times G_{2}$ has at least two vertices in $(S \times x) \cup(S \times y)$ at a distance 2 from it in $G_{1}\left[G_{2}\right]$. Thus $(S \times x) \cup(S \times y)$ is a DD-set in $G_{1}\left[G_{2}\right]$, proving that $\gamma_{2}^{d}\left(G_{1}\left[G_{2}\right]\right) \leq 2 \gamma_{2}^{d}\left(G_{1}\right)$.

Remark 3.6. 1. If $G_{1}$ has a universal vertex, but $G_{2}$ has no such vertex, then equality occurs in the above relation. 
2. If both $G_{1}$ and $G_{2}$ have a universal vertex then, strict inequality occurs in the above result.

3. If $G_{1}$ has a $\gamma_{2}^{d}$-set in which a pair of vertices are adjacent or if some vertex in $G_{1}$ is dominated by two different vertices in $S$, then strict inequality occurs in 3.5 .

Theorem 3.7. If $G_{1}$ has no isolated vertex, then for all graphs $G_{2}, \gamma_{2}^{d}\left(G_{1}\left[G_{2}\right]\right) \leq \gamma_{t}^{d}\left(G_{1}\right)$, where $\gamma_{t}^{d}\left(G_{1}\right)$ is the total disjunctive domination number of $G_{1}$.

Proof. Let $S$ be a TDD-set of $G_{1}$. For any vertex $x \in G_{2}$, we can show that $S \times x$ is a DD-set in $G_{1}\left[G_{2}\right]$. It is clear that $S \times x$ dominates or disjunctively dominates $\left(G_{1} \backslash S\right) \times G_{2}$. Now let $(u, v)$ be any vertex in $S \times x . u$ is either adjacent to $u^{\prime} \in S$ or has two vertices $u_{1}$ and $u_{2}$ in $S$ at a distance 2 from it. Then $(u, v)$ is either dominated by $\left(u^{\prime}, x\right) \in S \times x$ or disjunctively dominated by $\left(u_{1}, x\right),\left(u_{2}, x\right) \in S \times x$, showing that $S \times x$ is a disjunctive dominating set in $G_{1}\left[G_{2}\right]$. This proves that, $\gamma_{2}^{d}\left(G_{1}\left[G_{2}\right]\right) \leq \gamma_{t}^{d}\left(G_{1}\right)$.

Remark 3.8. The bound given in the above theorem is sharp. If $G_{1}$ has a universal vertex and $G_{2}$ has no such vertex, then $\gamma_{2}^{d}\left(G_{1}\left[G_{2}\right]\right)=\gamma_{t}^{d}\left(G_{1}\right)=2$. We may also note that strict inequality in the bound can be achieved. Consider the graphs $G_{1}=P_{5}, G_{2}=P_{2}$. Then $\gamma_{t}^{d}\left(G_{1}\right)=3, \gamma_{2}^{d}\left(G_{1}\left[G_{2}\right]=2\right.$ and hence $\gamma_{2}^{d}\left(G_{1}\left[G_{2}\right]\right)<\gamma_{t}^{d}\left(G_{1}\right)$.

\section{Disjunctive domination in tensor products}

Tensor product or Cross Product of graphs $G_{1}=\left(V_{1}, E_{1}\right)$ and $G_{2}=\left(V_{2}, E_{2}\right)$ is the graph $G_{1} \times G_{2}$ whose vertex set is $V_{1} \times V_{2}$ and edge set is $\left\{\left(\left(u_{1}, v_{1}\right),\left(u_{2}, v_{2}\right)\right): u_{1} u_{2} \in E_{1}\right.$ and $v_{1} v_{2} \in$ $\left.E_{2}\right\}$. There is no consistent relation between the disjunctive domination number of the tensor product of two graphs and the product of their disjunctive domination numbers. There are graphs in which $\gamma_{2}^{d}\left(G_{1} \times G_{2}\right)>\gamma_{2}^{d}\left(G_{1}\right) \gamma_{2}^{d}\left(G_{2}\right), \gamma_{2}^{d}\left(G_{1} \times\right.$ $\left.G_{2}\right)=\gamma_{2}^{d}\left(G_{1}\right) \gamma_{2}^{d}\left(G_{2}\right)$ and $\gamma_{2}^{d}\left(G_{1} \times G_{2}\right)<\gamma_{2}^{d}\left(G_{1}\right) \gamma_{2}^{d}\left(G_{2}\right)$.

Example 3.9. 1. $\gamma_{2}^{d}\left(P_{5} \times P_{3}\right)=4>\gamma_{2}^{d}\left(P_{5}\right) \gamma_{2}^{d}\left(P_{3}\right)$.

2. $\gamma_{2}^{d}\left(C_{3} \times C_{4}\right)=2=\gamma_{2}^{d}\left(C_{3}\right) \gamma_{2}^{d}\left(C_{4}\right)$.

3. If $G_{1}$ is the graph given in fig.1, then $\gamma_{2}^{d}\left(G_{1} \times G_{1}\right)=$ $2<\gamma_{2}^{d}\left(G_{1}\right) \gamma_{2}^{d}\left(G_{1}\right)$.

Theorem 3.10. For any two graphs $G_{1}$ and $G_{2}$ with at least two vertices and $G_{2}$ having no isolated vertices,

$$
\gamma_{2}^{d}\left(G_{1} \times G_{2}\right) \leq \min \left\{\gamma_{2}^{d}\left(G_{1}\right)\left|G_{2}\right|, \quad \gamma_{2}^{d}\left(G_{2}\right)\left|G_{1}\right|\right\}
$$

Proof. Let $G_{1}=\left(V_{1}, E_{1}\right)$ and $G_{2}=\left(V_{2}, E_{2}\right)$ are graphs with $\gamma_{2}^{d}$-sets $S_{1}$ and $S_{2}$ respectively. We can show that $S_{1} \times V_{2}$ and $V_{1} \times S_{2}$ are both $D D$-sets in $G_{1} \times G_{2}$.

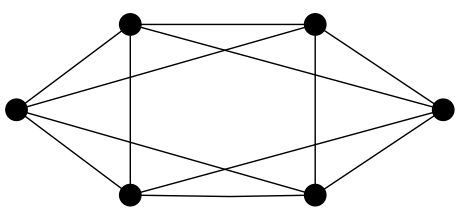

Figure 1. $G_{1}$

\section{claim}

Let $(u, v)$ be a vertex in $G_{1} \times G_{2}$.

If $u \in S_{1}$, then $(u, v) \in S_{1} \times V_{2}$. If $u \notin S_{1}$, then $u$ is either dominated by $x \in S_{1}$ or disjunctively dominated by two different vertices $x_{1}, x_{2} \in S_{1}$. If $u$ is dominated by $x \in S_{1}$, then the vertex $(u, v)$ in $G_{1} \times G_{2}$ is dominated by $\left(x, v^{\prime}\right) \in S_{1} \times V_{2}$, where $v^{\prime}$ is some vertex adjacent to $v$ in $G_{2}$. If $u$ is disjunctively dominated by $x_{1}, x_{2} \in S_{1}$, then the vertices $\left(x_{1}, v\right),\left(x_{2}, v\right) \in$ $S_{1} \times V_{2}$ are such that $d\left((u, v),\left(x_{1}, v\right)\right)=d\left((u, v),\left(x_{2}, v\right)\right)=2$. That is, $(u, v)$ has two vertices in $S_{1} \times V_{2}$ at a distance two from it. So, $(u, v) \in G_{1} \times G_{2}$ is disjunctively dominated by $S_{1} \times V_{2}$. Thus $S_{1} \times V_{2}$ is a $D D$-set of $G_{1} \times G_{2}$. Similarly, $V_{1} \times S_{2}$ is also a $D D$-set of $G_{1} \times G_{2}$. From these it follows that, $\gamma_{2}^{d}\left(G_{1} \times G_{2}\right) \leq \min \left\{\gamma_{2}^{d}\left(G_{1}\right)\left|G_{2}\right|, \gamma_{2}^{d}\left(G_{2}\right)\left|G_{1}\right|\right\}$.

Remark 3.11. 1. This bound is sharp. For example, if $G_{1}=P_{2}, G_{2}=P_{7}$, then $\gamma_{2}^{d}\left(G_{1}\right)=1, \gamma_{2}^{d}\left(G_{2}\right)=2$ and $\gamma_{2}^{d}\left(G_{1} \times G_{2}\right)=4$. In this case, $\gamma_{2}^{d}\left(G_{1} \times G_{2}\right)=\min \left\{\gamma_{2}^{d}\left(G_{1}\right)\left|G_{2}\right|, \gamma_{2}^{d}\left(G_{2}\right)\left|G_{1}\right|\right\}$

2. Strict inequality may occur in the above result. If $G_{1}=P_{3}$ and $G_{2}=P_{7}$, then $\gamma_{2}^{d}\left(G_{1}\right)=1, \gamma_{2}^{d}\left(G_{2}\right)=2$, $\gamma_{2}^{d}\left(G_{1} \times G_{2}\right)=5, \min \left\{\gamma_{2}^{d}\left(G_{1}\right)\left|G_{2}\right|, \gamma_{2}^{d}\left(G_{2}\right)\left|G_{1}\right|\right\}=6$. Here, $\gamma_{2}^{d}\left(G_{1} \times G_{2}\right)<\min \left\{\gamma_{2}^{d}\left(G_{1}\right)\left|G_{2}\right|, \gamma_{2}^{d}\left(G_{2}\right)\left|G_{1}\right|\right\}$.

\section{Disjunctive domination in strong products}

The strong product or normal product of graphs $G_{1}=$ $\left(V_{1}, E_{1}\right)$ and $G_{2}=\left(V_{2}, E_{2}\right)$ is the graph $G_{1} \otimes G_{2}$ whose vertex set is $V_{1} \times V_{2}$ in which $\left(u_{1}, v_{1}\right)$ is adjacent to $\left(u_{2}, v_{2}\right)$ if and only if either

- $u_{1}=u_{2}$ and $v_{1} v_{2} \in E_{2}$ or

- $u_{1} u_{2} \in E_{1}$ and $v_{1}=v_{2}$ or

- $u_{1} u_{2} \in E_{1}$ and $v_{1} v_{2} \in E_{2}$.

Theorem 3.12. For any two non trivial graphs $G_{1}$ and $G_{2}$,

$$
\gamma_{2}^{d}\left(G_{1} \otimes G_{2}\right) \leq \gamma_{2}^{d}\left(G_{1}\right) \gamma_{2}^{d}\left(G_{2}\right)
$$

Proof. Let $G_{1}=\left(V_{1}, E_{1}\right)$ and $G_{2}=\left(V_{2}, E_{2}\right)$ have $\gamma_{2}^{d}$-sets $S_{1}$ and $S_{2}$ respectively. We can show that $S_{1} \times S_{2}$ is a $D D$ - set of $G_{1} \otimes G_{2}$. 


\section{claim}

Let $(u, v) \notin S_{1} \times S_{2}$ be a vertex in $G_{1} \otimes G_{2}$.

\section{case (i)}

Let $u \in V_{1} \backslash S_{1}$ and $v \in S_{2}$. Then either $u$ is dominated by $x \in S_{1}$ or is disjunctively dominated by two different vertices $x_{1}, x_{2} \in$ $S_{1}$. If $u$ is dominated by $x \in S_{1}$, then $(u, v)$ is dominated by $(x, v) \in S_{1} \times S_{2}$ in $G_{1} \otimes G_{2}$. If $u$ is disjunctively dominated by two different vertices $x_{1}, x_{2} \in S_{1}$, then $\left(x_{1}, v\right),\left(x_{2}, v\right) \in S_{1} \times S_{2}$ and $d\left((u, v),\left(x_{1}, v\right)\right)=d\left((u, v),\left(x_{2}, v\right)\right)=2$ so that $(u, v)$ is disjunctively dominated by $S_{1} \times S_{2}$ in $G_{1} \otimes G_{2}$.

\section{case (ii)}

Let $u \in V_{1}$ and $v \in V_{2} \backslash S_{2}$. Then either $v$ is dominated by $y \in S_{2}$ or is disjunctively dominated by two different vertices $y_{1}, y_{2} \in S_{2}$. If $v$ is dominated by $y \in S_{2},(u, v)$ is dominated by $(u, y) \in S_{1} \times S_{2}$ in $G_{1} \otimes G_{2}$. If $v$ is disjunctively dominated by two vertices $y_{1}, y_{2} \in S_{2}$, then $\left(u, y_{1}\right),\left(u, y_{2}\right) \in S_{1} \times S_{2}$ and $d\left((u, v),\left(u, y_{1}\right)\right)=d\left((u, v),\left(u, y_{2}\right)\right)=2$ so that $(u, v)$ is disjunctively dominated by $S_{1} \times S_{2}$ in $G_{1} \otimes G_{2}$.

\section{case (iii)}

Let $u \in V_{1} \backslash S_{1}$ and $v \in V_{2} \backslash S_{2}$. If $u$ is dominated by $x \in S_{1}$ and $v$ is dominated by $y \in S_{2}$, then $(u, v)$ is dominated by $(x, y) \in S_{1} \times S_{2}$ in $G_{1} \otimes G_{2}$.

If $u$ is disjunctively dominated by two different vertices $x_{1}, x_{2} \in S_{1}$ in $G_{1}$ and $v$ is dominated by $y \in S_{2}$ in $G_{2}$, then $(u, v)$ is adjacent to $\left(u_{1}, y\right)$ which is again adjacent to $\left(x_{1}, y\right) \in$ $S_{1} \times S_{2}$. Similarly, $(u, v)$ is also adjacent to $\left(u_{2}, y\right)$ which is again adjacent to $\left(x_{2}, y\right) \in S_{1} \times S_{2}$. Thus $d\left((u, v),\left(x_{1}, y\right)\right)=$ $d\left((u, v),\left(x_{2}, y\right)\right)=2$. In other words $(u, v)$ is disjunctively dominated by two different vertices $\left(x_{1}, y\right),\left(x_{2}, y\right) \in S_{1} \times S_{2}$. Similarly if $u$ is dominated by $x \in S_{1}$ in $G$ and $v$ is disjunctively dominated $y_{1}, y_{2} \in S_{2}$ in $G_{2}$, then $(u, v)$ is disjunctively dominated by $\left(x, y_{1}\right),\left(x . y_{2}\right) \in S_{1} \times S_{2}$ in $G_{1} \otimes G_{2}$.

If $u$ and $v$ are both disjunctively dominated by $S_{1}$ in $G_{1}$ and $S_{2}$ in $G_{2}$ respectively, then there exist $x_{1}, x_{2} \in S_{1}$ and $y_{1}, y_{2} \in S_{2}$ such that $d\left(u, x_{1}\right)=d\left(u, x_{2}\right)=2$ in $G_{1}$ and $d\left(v, y_{1}\right)=d\left(v, y_{2}\right)=2$ in $G_{2}$. Then there exist $u_{1}, u_{2} \in V_{1} \backslash S_{1}$ such that $u$ is adjacent to $u_{1}$ and $u_{2}$ where $u_{1}, u_{2}$ are respectively adjacent to $x_{1}$ and $x_{2}$ in $G$. Similarly, there exist $v_{1}, v_{2} \in V_{2} \backslash S_{2}$ such that $v$ is adjacent to $v_{1}$ and $v_{2}$ where $v_{1}, v_{2}$ are respectively adjacent to $y_{1}$ and $y_{2}$ in $G_{2}$. Thus in $G_{1} \otimes G_{2}$, vertex $(u, v)$ is adjacent to $\left(u_{1}, v_{1}\right)$ and $\left(u_{2}, v_{2}\right)$ which are respectively adjacent to $\left(x_{1}, y_{1}\right)$ and $\left(x_{2}, y_{2}\right)$ in $S_{1} \times S_{2}$. Then, $d\left((u, v),\left(x_{1}, y_{1}\right)\right)=d\left((u, v),\left(x_{2}, y_{2}\right)\right)=2$, proving that $(u, v)$ is disjunctively dominated by $S_{1} \times S_{2}$.

The above cases show that $S_{1} \times S_{2}$ is a $D D$-set in $G_{1} \otimes G_{2}$. Thus $\gamma_{2}^{d}\left(G_{1} \otimes G_{2}\right) \leq \gamma_{2}^{d}\left(G_{1}\right) \gamma_{2}^{d}\left(G_{2}\right)$.

Remark 3.13. $\quad$ 1. The above bound is sharp. For example if $G_{1}=P_{2}$ and $G_{2}=P_{7}$, then $\gamma_{2}^{d}\left(G_{1}\right)=1, \gamma_{2}^{d}\left(G_{2}\right)=2$, $\gamma_{2}^{d}\left(G_{1} \otimes G_{2}\right)=2$. So $\gamma_{2}^{d}\left(G_{1} \otimes G_{2}\right)=\gamma_{2}^{d}\left(G_{1}\right) \gamma_{2}^{d}\left(G_{2}\right)$.

2. Strict inequality occurs if $G_{1}=G_{2}=P_{4}$. Then $\gamma_{2}^{d}\left(G_{1}\right)=$ $\gamma_{2}^{d}\left(G_{2}\right)=2$ and $\gamma_{2}^{d}\left(G_{1} \otimes G_{2}\right)=2$. Hence, $\gamma_{2}^{d}\left(G_{1} \otimes\right.$ $\left.G_{2}\right)<\gamma_{2}^{d}\left(G_{1}\right) \gamma_{2}^{d}\left(G_{2}\right)$.

\section{Disjunctive domination in cartesian products}

The Cartesian Product $G_{1} \square G_{2}$ of graphs $G_{1}=\left(V_{1}, E_{1}\right)$ and $G_{2}=\left(V_{2}, E_{2}\right)$ is the graph with vertex set $V_{1} \times V_{2}$ in which $\left(u_{1}, v_{1}\right),\left(u_{2}, v_{2}\right)$ is an edge if and only if either

- $u_{1}=u_{2}$ and $v_{1} v_{2} \in E_{2}$ or

- $u_{1} u_{2} \in E_{1}$ and $v_{1}=v_{2}$

Theorem 3.14. For any two graphs $G_{1}$ and $G_{2}$,

$$
\gamma_{2}^{d}\left(G_{1} \square G_{2}\right) \leq \min \left\{\gamma_{2}^{d}\left(G_{1}\right)\left|G_{2}\right|, \gamma_{2}^{d}\left(G_{2}\right)\left|G_{1}\right|\right\}
$$

Proof. Let $G_{1}$ and $G_{2}$ are two graphs with $\gamma_{2}^{d}$-sets $S_{1}$ and $S_{2}$ respectively. We can show that $S_{1} \times V_{2}$ and $V_{1} \times S_{2}$ are both $D D$-sets of $G_{1} \square G_{2}$.

\section{claim}

Let $(u, v)$ be a vertex in $G_{1} \square G_{2}$. If $u \in S_{1}$, then $(u, v) \in$ $S_{1} \times V_{2}$. If $u \notin S_{1}$, then $u$ is either dominated by $x \in S_{1}$ or disjunctively dominated by two different vertices $x_{1}, x_{2} \in$ $S_{1}$. If $u$ is dominated by $x \in S_{1}$, then $(u, v)$ is adjacent to $(x, v) \in S_{1} \times V_{2}$. If $u$ is disjunctively dominated by $x_{1}, x_{2} \in$ $S_{1}$, then the vertices $\left(x_{1}, v\right),\left(x_{2}, v\right) \in S_{1} \times V_{2}$ are such that $d\left((u, v),\left(x_{1}, v\right)\right)=d\left((u, v),\left(x_{2}, v\right)\right)=2$. That is, $(u, v)$ has two vertices in $S_{1} \times V_{2}$ at a distance two from it. Thus it is disjunctively dominated by $S_{1} \times V_{2}$. Hence $S_{1} \times V_{2}$ is a $D D$ set of $G_{1} \square G_{2}$. Similarly, $V_{1} \times S_{2}$ is also a $D D$-set of $G_{1} \square G_{2}$. Thus $\gamma_{2}^{d}\left(G_{1} \square G_{2}\right) \leq \min \left\{\gamma_{2}^{d}\left(G_{1}\right)\left|G_{2}\right|, \gamma_{2}^{d}\left(G_{2}\right)\left|G_{1}\right|\right\}$.

Remark 3.15. 1. Equality comes in the above theorem if $G_{1}=P_{2}$ or $P_{3}$ and $G_{2}=P_{2}$.

2. Strict inequality occurs if $G_{1}=P_{2}$ and $G_{2}=P_{7}$.

Remark 3.16. The Vizing's like inequality $\gamma_{2}^{d}\left(G_{1} \square G_{2}\right) \geq$ $\gamma_{2}^{d}\left(G_{1}\right) \gamma_{2}^{d}\left(G_{2}\right)$ is not true in disjunctive domination. There are graphs in which $\gamma_{2}^{d}\left(G_{1} \square G_{2}\right)>\gamma_{2}^{d}\left(G_{1}\right) \gamma_{2}^{d}\left(G_{2}\right), \gamma_{2}^{d}\left(G_{1} \square G_{2}\right)=$ $\gamma_{2}^{d}\left(G_{1}\right) \gamma_{2}^{d}\left(G_{2}\right)$ and $\gamma_{2}^{d}\left(G_{1} \square G_{2}\right)<\gamma_{2}^{d}\left(G_{1}\right) \gamma_{2}^{d}\left(G_{2}\right)$.

For example,

1. If $G_{1}=P_{7}$ and $G_{2}=P_{2}$, then $\gamma_{2}^{d}\left(G_{1} \square G_{2}\right)=3>\gamma_{2}^{d}\left(G_{1}\right) \gamma_{2}^{d}\left(G_{2}\right)$.

2. If $G_{1}=C_{4}$ and $G_{2}=P_{2}$, then $\gamma_{2}^{d}\left(G_{1} \square G_{2}\right)=\gamma_{2}^{d}\left(G_{1}\right) \gamma_{2}^{d}\left(G_{2}\right)=2$.

3. If $G_{1}=G_{2}=C_{4}$, then $\gamma_{2}^{d}\left(G_{1}\right)=\gamma_{2}^{d}\left(G_{2}\right)=2$ and $\gamma_{2}^{d}\left(G_{1} \square G_{2}\right)=2$. Hence $\gamma_{2}^{d}\left(G_{1} \square G_{2}\right)<\gamma_{2}^{d}\left(G_{1}\right) \gamma_{2}^{d}\left(G_{2}\right)$.

Theorem 3.17. For any two graphs $G_{1}$ and $G_{2}$, where $G_{1}$ has a $\gamma$ - set which is such that the vertices not in this set are twice dominated, $\gamma_{2}^{d}\left(G_{1} \square G_{2}\right) \leq \gamma\left(G_{1}\right) \gamma\left(G_{2}\right)$.

Proof. Let $G_{1}=\left(V_{1}, E_{1}\right)$ and $G_{2}=\left(V_{2}, E_{2}\right)$ be two graphs with $\gamma$ - sets $S_{1}$ and $S_{2}$ respectively. Let the elements of $V_{1} \backslash S_{1}$ are dominated by two different vertices in $S_{1}$. We can show that $S_{1} \times S_{2}$ is a disjunctive dominating set of $G_{1} \square G_{2}$. Let $(u, v)$ be a vertex in $G_{1} \square G_{2}$. 
case (i)

If $u \in S_{1}$ and $v \in S_{2}$, then $(u, v) \in S_{1} \times S_{2}$.

\section{case (ii)}

Let $u \in S_{1}$ and $v \in V_{2} \backslash S_{2}$. If $v$ is dominated by $x \in S_{2}$ in $G_{2}$, then $(u, v)$ is dominated by $(u, x) \in S_{1} \times S_{2}$ in $G_{1} \square G_{2}$. Similar is the case when $u \in V_{1} \backslash S_{1}$ and $v \in S_{2}$.

\section{case (iii)}

Let $u \in V_{1} \backslash S_{1}$ and $v \in V_{2} \backslash S_{2}$. By hypothesis $u$ is adjacent to two different vertices $x_{1}, x_{2} \in S_{1}$ in $G_{1}$ and $v$ is adjacent to $y \in S_{2}$ in $G_{2}$. Then in $G_{1} \square G_{2},(u, v)$ is adjacent to $(u, y)$ which is adjacent to $\left(x_{1}, y\right)$ and $\left(x_{2}, y\right) \in S_{1} \times S_{2}$. Thus there are two different vertices $\left(x_{1}, y\right),\left(x_{2}, y\right) \in S_{1} \times S_{2}$ such that $d\left((u, v),\left(x_{1}, y\right)\right)=d\left((u, v),\left(x_{2}, y\right)\right)=2$. Hence $(u, v)$ is disjunctively dominated by $S_{1} \times S_{2}$.

The above cases show that $S_{1} \times S_{2}$ is a disjunctive dominating set of $G_{1} \square G_{2}$. Hence $\gamma_{2}^{d}\left(G_{1} \square G_{2}\right) \leq \gamma\left(G_{1}\right) \gamma\left(G_{2}\right)$.

Remark 3.18. The above result is not true in general. The following examples show this.

1. If $G_{1}=G_{2}=P_{6}, \gamma\left(G_{1}\right)=\gamma_{2}^{d}\left(G_{1}\right)=2, \gamma\left(G_{2}\right)=\gamma_{2}^{d}\left(G_{2}\right)=$ $2, \gamma_{2}^{d}\left(G_{1} \square G_{2}\right)=6>\gamma_{2}^{d}\left(G_{1}\right) \gamma_{2}^{d}\left(G_{2}\right)=\gamma\left(G_{1}\right) \gamma\left(G_{2}\right)$.

2. If $G_{1}=G_{2}=P_{7}, \gamma\left(G_{1}\right)=\gamma\left(G_{2}\right)=3, \gamma_{2}^{d}\left(G_{1}\right)=\gamma_{2}^{d}\left(G_{2}\right)=$ 2, $\gamma_{2}^{d}\left(G_{1} \square G_{2}\right)=8, \gamma_{2}^{d}\left(G_{1}\right) \gamma_{2}^{d}\left(G_{2}\right)<\gamma_{2}^{d}\left(G_{1} \square G_{2}\right)<$ $\gamma\left(G_{1}\right) \gamma\left(G_{2}\right)$.

3. If $G_{1}=G_{2}=P_{10}, \gamma\left(G_{1}\right)=\gamma\left(G_{2}\right)=4, \gamma_{2}^{d}\left(G_{1}\right)=\gamma_{2}^{d}\left(G_{2}\right)=$ $3, \gamma_{2}^{d}\left(G_{1} \square G_{2}\right)=15$. Here, $\gamma_{2}^{d}\left(G_{1}\right) \gamma_{2}^{d}\left(G_{2}\right)<\gamma_{2}^{d}\left(G_{1} \square G_{2}\right)<$ $\gamma\left(G_{1}\right) \gamma\left(G_{2}\right)$.

4. If $G_{1}=G_{2}=P_{11}, \gamma_{2}^{d}\left(G_{1}\right)=\gamma_{2}^{d}\left(G_{2}\right)=3, \gamma\left(G_{1}\right)=\gamma\left(G_{2}\right)=$ $4, \gamma_{2}^{d}\left(G_{1} \square G_{2}\right)=18$. Here $\gamma_{2}^{d}\left(G_{1}\right) \gamma_{2}^{d}\left(G_{2}\right)<\gamma\left(G_{1}\right) \gamma\left(G_{2}\right)<$ $\gamma\left(G_{1} \square G_{2}\right)$.

Theorem 3.19. For any two positive integers $m, n, \gamma_{2}^{d}\left(K_{m} \square K_{n}\right)=$ 2.

Proof. Let $\left(u_{1}, v_{1}\right),\left(u_{2}, v_{2}\right)$ are two distinct vertices in $K_{m} \square K_{n}$. A vertex $(x, y) \in K_{m} \square K_{n}$ which not dominated by these vertices is such that $d\left(\left(u_{1}, v_{1}\right),(x, y)\right)=d\left(\left(u_{2}, v_{2}\right),(x, y)\right)=2$. Hence $\left\{\left(u_{1}, v_{1}\right),\left(u_{2}, v_{2}\right)\right\}$ is a $D D$-set in $K_{m} \square K_{n}$ which gives $\gamma_{2}^{d}\left(K_{m} \square K_{n}\right) \leq 2$. If $u_{1} \neq u_{2}$ and $v_{1} \neq v_{2}$ then $\left(u_{1}, v_{1}\right)$ and $\left(u_{2}, v_{2}\right)$ are not adjacent in $K_{m} \square K_{n}$. So there does not exist a universal vertex in $K_{m} \square K_{n}$ which implies that $\gamma_{2}^{d}\left(K_{m} \square K_{n}\right) \geq$ 2. Therefore $\gamma_{2}^{d}\left(K_{m} \square K_{n}\right)=2$.

\section{Conclusion}

In this paper we have tried to find properties of disjunctive domination in certain product of graphs. Further investigations are possible to find $D D$-number of product of important classes of graphs. The problem of determining $\gamma_{2}^{d}\left(G_{1} * G_{2}\right)$ precisely for different classes of graphs would be interesting.

\section{Acknowledgment}

The first author wishes to thank St. Mary's College, Thrissur and Univerity of Calicut, Kerala for providing necessary facilities to pursue her research.

\section{References}

[1] F. Harary, Graph Theory, Addison-Wesley Publishing Co., Reading, MA/Menlo Park, CA/London, 1969.

[2] Teresa W. Haynes, Stephen T. Hedetniemi, Peter J.Slater, Fundamentals of Domination in Graphs, Marcel Dekker, New York, 1998.

[3] Sandra M Hedetniemi, Stephen T. Hedetniemi, J Knisely, D. F Rall, Secondary domination in graphs, $A K C E J$. Graphs Comb., 5(2008), 103-115.

[4] Wayne Goddard, M. A Henning, Charles A McPillan, The Disjunctive Domination Number of a Graph, Quaest. Math., 37(4)(2014), 547-561.

[5] M.A Henning, Sinclair A Marcon, Domination versus Disjunctive Domination in Graphs, Quaest. Math., 2015, $1-13$.

[6] M. A Henning, Viroshan Naicker, Bounds on the Disjunctive Total Domination number of a Tree, Discussions Mathematicae Graph Theory, 36(2016), 153-171.

[7] R. Hammack, W. Imrich, S. Klavzar, Handbook of Product Graphs, Discrete Mathematics and its Applications, CRC Press, Second Edition, 2011.

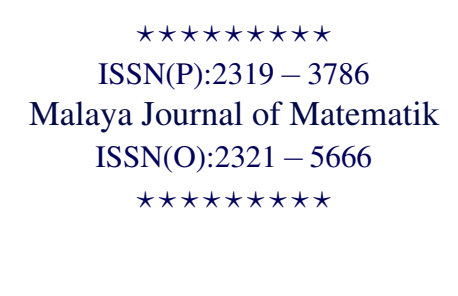

